\title{
Les Syndromes Drépanocytaires Majeurs Chez L'enfant et L'adolescent: Étude de la Cohorte du Centre Hospitalier Régional de Saint-Louis (Sénégal)
}

\author{
Ndiogou Seck, \\ Khadim Bop, \\ Oumy Mbacke, \\ Ibrahima Diagne
}

Service de pédiatrie, Centre Hospitalier Régional de Saint-Louis, boulevard Abdoulaye Mar Diop, Saint-Louis, Sénégal

\section{Lamine Thiam}

Service de Pédiatrie, Hôpital de la Paix, Boucoutte, Ziguinchor, Sénégal

\section{Aliou Abdoulaye Ndongo,}

Younoussa Keïta

Service de pédiatrie Hôpital Aristide Ledantec,Dakar, Sénégal

Idrissa Basse

Hôpital pour enfants de Diamniadio, Bargny, Sénégal

\section{Djibril Boiro,}

Amadou Sow

Service de pédiatrie, Centre Hospitalier Abass Ndao, Avenue Cheikh Anta

Diop, Dakar, Sénégal

Doi:10.19044/esj.2021.v17n34p70

Submitted: 21 June 2021

Accepted: 23 August 2021

Published: 30 September 2021
Copyright 2021 Author(s)

Under Creative Commons BY-NC-ND 4.0 OPEN ACCESS

Cite As:

Seck N., Bop K., Mbacke O., Diagne I., Thiam L., Ndongo A.A., Keïta Y., Basse I., Boiro D. \& Sow A. (2021). Les Syndromes Drépanocytaires Majeurs Chez L'enfant et L'adolescent : Étude de la Cohorte du Centre Hospitalier Régional de Saint-Louis (Sénégal). European Scientific Journal, ESJ, 17(34), 70.

https://doi.org/10.19044/esj.2021.v17n34p70

\section{Résumé}

La drépanocytose est une maladie héréditaire de l’hémoglobine, à transmission autosomique récessive, caractérisée par la présence d’une hémoglobine anormale appelée " hémoglobine S ». Les syndromes drépanocytaires majeurs regroupent la forme homozygote "SS » et les hétérozygoties composites ( $\mathrm{SC}, \mathrm{SD}$ et $\mathrm{S}$ béta thalassémie « $\mathrm{S} \beta$ »). Depuis 
février 2011, une cohorte d'enfants et d'adolescents atteints de syndrome drépanocytaire majeur est suivi au Centre Hospitalier Régional de Saint-Louis, Sénégal. Cette cohorte a fait l'objet de notre travail dont l'objectif principal était de décrire les caractéristiques épidémiologiques, cliniques, biologiques et évolutifs.

Il s'agissait d'une étude épidémiologique, rétrospective et descriptive portant sur l'ensemble des dossiers des patients âgés de 0 à 20 ans, porteurs d'un syndrome drépanocytaire majeur, régulièrement suivis à la période du 1er janvier 2011 au 31 décembre 2016 (6 ans).

Le diagnostic de syndrome drépanocytaire majeur était établi sur la base d'une NFS et d'une électrophorèse de l'hémoglobine.

Durant la période d'étude 191 patients porteurs d'un syndrome drépanocytaire majeur ont été suivis au Centre Hospitalier Régional de SaintLouis, Sénégal. Cent vingt-sept (127) dossiers ont été retenus dont 71 garçons et 56 filles. La majorité des patients (111 soit 87\%) étaient de profil homozygote «SS ». L'âge moyen au diagnostic de la maladie était de 4 ans et 10 mois et l'âge moyen de début du suivi était de 6 ans 2 mois. Les circonstances de découverte de la maladie étaient dominées par une crise vasoocclusive (58,5 \% des cas). Le taux d'hémoglobine de base moyen était de 8,6 $\mathrm{g} / \mathrm{dl}$. Les deux principales complications aiguës rencontrées étaient une anémie aigue (22 \%) et les infections sévères (22 \%). Les complications chroniques étaient dominées par la lithiase vésiculaire (5,5\%). Le nombre moyen d'hospitalisation était de 1,9. La létalité était de 3,1 \%.

La drépanocytose est fréquente au Sénégal. Elle est relativement bien tolérée malgré son diagnostic et sa prise en charge tardifs. Pour améliorer la prise en charge et le pronostic des malades, il sera nécessaire de mettre en place un programme national de dépistage néonatal et la création de centres de référence de prise en charge et de suivi des patients drépanocytaires.

Mots clés: Syndrome Drépanocytaire Majeur, Morbidité, Enfant, Adolescent, Sénégal 


\title{
Sickle Cell Disease in Children and Adolescents at Saint-Louis Regional Hospital (Senegal)
}

\author{
Ndiogou Seck, \\ Khadim Bop, \\ Oumy Mbacke, \\ Ibrahima Diagne \\ Service de pédiatrie, Centre Hospitalier Régional de Saint-Louis, boulevard \\ Abdoulaye Mar Diop, Saint-Louis, Sénégal \\ Lamine Thiam \\ Service de Pédiatrie, Hôpital de la Paix, Boucoutte, Ziguinchor, Sénégal \\ Aliou Abdoulaye Ndongo, \\ Younoussa Keïta \\ Service de pédiatrie Hôpital Aristide Ledantec,Dakar, Sénégal \\ Idrissa Basse \\ Hôpital pour enfants de Diamniadio, Bargny, Sénégal \\ Djibril Boiro, \\ Amadou Sow \\ Service de pédiatrie, Centre Hospitalier Abass Ndao, Avenue Cheikh Anta \\ Diop, Dakar, Sénégal
}

Abstract

Sickle cell disease is an inherited disease of hemoglobin, with recessive autosomal transmission, characterized by the presence of an abnormal hemoglobin called hemoglobin "S". Major sickle cell syndromes include the homozygote form "SS" and composite heterozygous (SC, SD and S beta thalassemia). Since February 2011, a cohort of children and adolescents with sickle cell disease has been monitored at the St. Louis Regional Hospital Center, Senegal. This cohort was the focus of our work, the main objective of which was to describe epidemiological, clinical, biological, and evolutionary characteristics.

This was an epidemiological, retrospective, and descriptive study of all the records of patients aged 0 to 20 years, with major sickle cell syndrome, regularly followed in the period from 1 January 2011 to 31 December 2016 (6 years).

The diagnosis of major sickle cell syndrome was based on hemogram and hemoglobin electrophoresis.

During the study period 191 patients with major sickle cell syndrome were followed at St. Louis Regional Hospital Center, Senegal. One hundred and twenty-seven (127) were retained, including 71 boys and 56 girls. Most patients (111 or $87 \%$ ) were of homozygotic "SS" profile. The average age at diagnosis of the disease was 4 years and 10 months and the average age of 
onset of follow-up was 6 years 2 months. The circumstances of discovery of the disease were dominated by a vaso-occlusive crisis (58.5\% of cases). The average baseline hemoglobin level was $8.6 \mathrm{~g} / \mathrm{dL}$. The two main acute complications encountered were acute anaemia (22\%) and severe infections (22\%). Chronic complications were dominated by vesicular lithiasis (5.5\%). The average number of hospitalizations was 1.9 . The lethality was $3.1 \%$.

Sickle cell disease is common in Senegal. It is relatively well tolerated despite its late diagnosis and management. To improve the management and prognosis of patients, it will be necessary to set up a national neonatal screening program and the creation of referral centers for the management and follow-up of sickle cell patients.

Keywords: Major Sickle Cell Syndrome, Morbidity, Child, Adolescent, Senegal

\section{Introduction}

La drépanocytose est une maladie héréditaire de l'hémoglobine, à transmission autosomique récessive, caractérisée par la présence d'une hémoglobine anormale appelée "hémoglobine $\mathrm{S}$ ». Elle résulte d'une substitution de la valine par l'acide glutamique en position six de la chaine béta de globine. C’est la maladie génétique la plus répandue dans le monde. Elle touche essentiellement mais pas exclusivement les populations de race Noire (Bardakjian \& Wajcman, 2004).

Elle se présente sous deux formes: la forme hétérozygote «AS » ou trait drépanocytaire et les syndromes drépanocytaires majeurs qui regroupent la forme homozygote " SS » et les hétérozygoties composites (SC, SD et S béta thalassémie « S $\beta$ ») (Bardakjian \& Wajcman, 2004).

Dans le monde, selon Loupec (2014), près de 120 millions de personnes seraient porteuses d'une mutation drépanocytaire et chaque année, 500000 nouvelles naissances seraient concernées. Au Sénégal $10 \%$ de la population générale serait atteinte et chaque année, près de 1700 nouveau-nés (soit $0,5 \%$ des naissances) naissent avec la forme homozygote « SS » (Boiro et al., 2016), (North et al., 1995).

La drépanocytose est une maladie chronique dont l'évolution peut être émaillée de complications aiguës (anémie aigue, infections graves, accidents vaso-occlusif majeurs) et chroniques (lithiase biliaire, ostéonécrose aseptique de la tête fémorale, insuffisance cardiaque, insuffisance rénale...).

Ces complications sont à l'origine d'une létalité non négligeable et d'une importante morbidité surtout dans les pays en développement. En effet, la mortalité infanto-juvénile des sujets porteurs de syndromes drépanocytaires majeurs constitue l’une des plus élevées dans la population générale. 
Depuis février 2011, une cohorte d'enfants et d'adolescents atteints de syndrome drépanocytaire majeur est suivi au Centre Hospitalier Régional de Saint-Louis (CHR/SL), Sénégal. Cette cohorte a fait l'objet de notre travail dont :

L’objectif principal était: de décrire les caractéristiques épidémiologiques, cliniques et évolutifs des enfants et adolescents suivis pour syndrome drépanocytaire majeur au CHR/SL

Les objectifs spécifiques étaient de:

- décrire les caractéristiques socio démographiques des enfants et adolescents porteurs de syndrome drépanocytaire majeur ;

- décrire les aspects cliniques et biologiques des enfants et adolescents porteurs de syndrome drépanocytaire majeur ;

- analyser l'influence des aspects épidémio-cliniques sur l'évolution de la maladie.

\section{Patients et méthodes}

Notre travail s'est déroulé dans le service de pédiatrie du Centre Hospitalier Régional de Saint-Louis. C'est l'unique établissement de la région qui propose depuis février 2011 une prise en charge et un suivi des enfants et adolescents atteints de syndrome drépanocytaire majeur.

Il s'agissait d'une étude épidémiologique, rétrospective et descriptive portant sur l'ensemble des dossiers des patients âgés de 0 à 20 ans, porteurs d'un syndrome drépanocytaire majeur, régulièrement suivis à la période du $1^{\text {er }}$ janvier 2011 au 31 décembre 2016 (6 ans).

Le diagnostic de syndrome drépanocytaire majeur était établi sur la base d'une numération formule sanguine (NFS) et d'une électrophorèse de l'hémoglobine.

Une fois le diagnostic retenu, les patients étaient revus systématiquement en consultation tous les trois à quatre mois en l'absence de manifestations critiques ou d'affections intercurrentes. Cependant les patients présentant des manifestations aigues étaient admis en urgence dans le service de pédiatrie.

\section{Critères d'inclusion}

Les dossiers médicaux de tous les enfants et adolescents âgés de 0 à 20 ans atteints de syndrome drépanocytaire majeur, suivis régulièrement durant la période d'étude. 


\section{Critères de non inclusion}

Les dossiers ne pouvant pas être exploités en raison des données manquantes et les dossiers de patients perdus de vue sur une période de plus de 12 mois.

\section{Recueil et analyses des données}

Les données étaient collectées par l'étude des dossiers médicaux et consignées sur une fiche d'enquête individuelle, elles étaient éventuellement complétées au téléphone si la famille est joignable.

Les paramètres étudiés ont été :

- Les données sociodémographiques : âge, sexe, taille de la fratrie, niveau d'instruction et niveau socio-économique des parents, degré de consanguinité, statut vaccinal des patients...

- Les données anamnestiques et cliniques : âge et circonstances de découverte de la maladie, le nombre de crises vaso-occlusives...

- Les données biologiques: type d'hémoglobinopathie, le taux d'hémoglobine de base...

- Les données évolutives : le nombre d'hospitalisations de transfusion sanguine, les complications aigues et chroniques...

Ces données ont été saisies et analysées avec le logiciel Epi info version 3.5.3 (Référence).. Le seuil de significativité retenu est une valeur de $\mathrm{p}$ inférieure à 0,05 .

\section{Résultats}

Du $1^{\mathrm{er}}$ janvier 2011 au 31 décembre 2016, 191 patients porteurs d'un syndrome drépanocytaire majeur ont été suivis au CHR/SL. Cent vingt-sept (127) dossiers ont pu être exploités et retenus pour l'étude. Le sexe ratio était de 1,2 avec 71 garçons.

Sur le plan génotypique, la majorité des patients (111 soit $87 \%$ ) étaient de profil homozygote "SS » et 10 (8\%) étaient des hétérozygotes «SC » (figure 1).

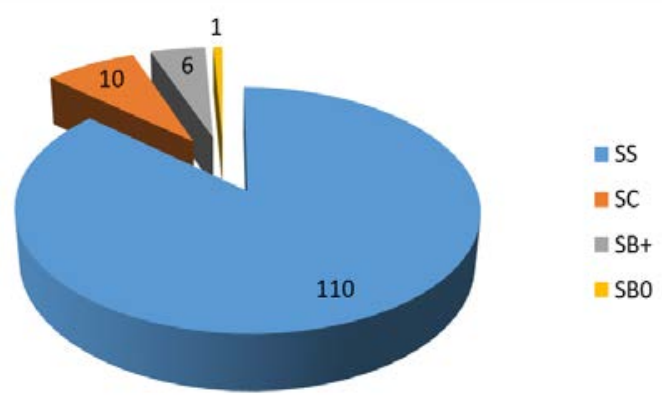

Figure 1. Profil génotypique des patients drépanocytaires suivis au CHR 
L'âge des patients au moment de l'étude variait entre 2 ans 5 mois et 19 ans 11 mois avec une moyenne de 10 ans 6 mois. La majorité des patients (91,4 \%) avaient plus de 5 ans (figure 2).

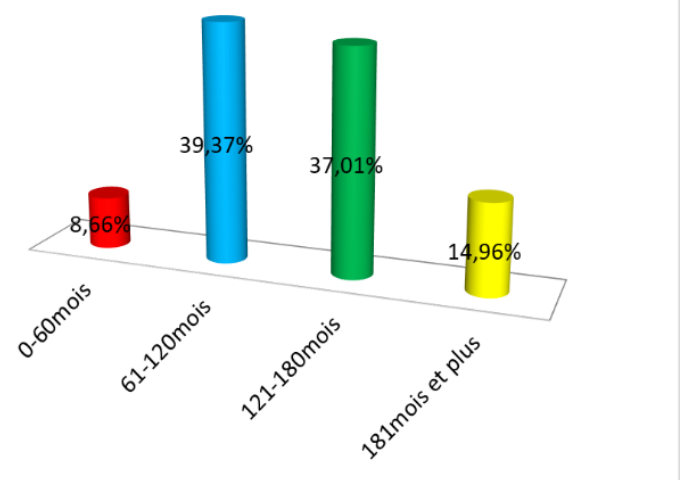

Figure 2. Répartition des patients en tranches d'âge de 60 mois

L'âge au diagnostic de la maladie survenait entre 1 mois et 17 ans 7 mois avec une moyenne de 4 ans 10 mois. Chez $60 \%$ des patients, la maladie a été découverte au cours des cinq premières années de vie.

L'âge de début du suivi de la maladie était de 7 mois à 17 ans 8 mois avec une moyenne de 6 ans 2 mois. Près de la moitié (44,7 \%) des patients avaient démarré leur suivi avant l'âge de 5 ans.

Le niveau de scolarisation a été renseigné pour 105 patients, seuls 8 (7,6 \%) des enfants n'étaient pas scolarisés.

Concernant le niveau d'instruction des parents, seuls $12,3 \%$ des pères et $4,3 \%$ des mères avaient reçu une instruction jusqu'au niveau secondaire correspondant au lycée. Le taux d'analphabétisme était plus faible chez les pères $(2,2 \%)$ vs $11,9 \%$ chez les mères.

La majorité des patients (80 \%) étaient issus de ménages sans ou à faibles revenus.

Il existait un lien de consanguinité parentale dans près de la moitié (51,3 \%) des unions. Cette consanguinité était apparentée au deuxième degrés dans deux tiers des cas.

Le statut vaccinal des patients a été apprécié en premier par la réalisation des vaccins du programme élargi de vaccination (PEV) qui permet, de la naissance à 15 mois, une protection contre la tuberculose, l'hépatite B, la diphtérie, le tétanos, la coqueluche, la poliomyélite, les infections à pneumocoque, à Haemophilus influenzae b, à rotavirus, la fièvre jaune, la rougeole et la rubéole. Puis par la réalisation de vaccins fortement recommandés chez le patient drépanocytaire. La majorité des patients (111 soit 87,4 \%) étaient à jour pour le PEV. Quant aux vaccins recommandés, la 
couverture était de 25,4 \% pour le pneumocoque, 44 \% par rapport à la fièvre typhoïde et 26,7 \% pour les infections à méningocoques (A,C,Y et W135).

Les circonstances de la découverte de la maladie ont été précisées chez 123 patients. Chez la majorité des patients (114 soit 92,6 \%), la drépanocytose a été découverte lors des manifestations de la maladie notamment à l'occasion d'une crise vaso-occlusive (CVO) dans 58,5 \% des cas, d'une anémie dans $21,1 \%$ des cas et chez 14 patients (11,3\%) à l'occasion d'une complication aigue de la maladie (figure 3 ).

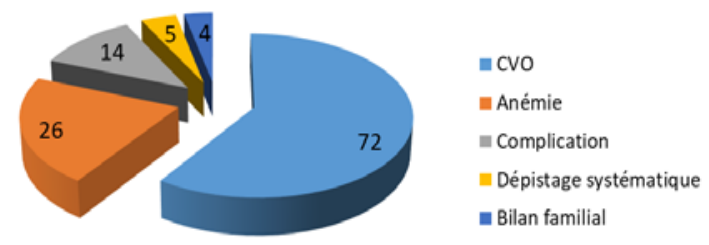

Figure 3. Répartition des patients selon les circonstances de découverte de la maladie $\mathrm{CVO}$ : crise vaso-occlusive

Dans notre cohorte, sur les 127 patients, 30 (24\%) n'avaient jamais été hospitalisés. Chez les 95 autres, le nombre d'hospitalisation variait de 1 à 10 avec une moyenne de 1,9. la durée des hospitalisations variait de 2 à 63 jours avec une moyenne de 9,5 jours.

Les complications aigues rencontrées chez nos patients tout au long du suivi en fonction du type de drépanocytose ont été résumées dans le tableau I.

Tableau I. Fréquence des complications aiguës en fonction du profil génotypique des patients

\begin{tabular}{|c|c|c|c|c|}
\hline Drofil & SS & $\overline{\mathrm{SC}}$ & SB plus & SBzéro \\
\hline Anémie aiguë & 26 & 00 & 1 & 1 \\
\hline Accident & 7 & 00 & 00 & 00 \\
\hline $\begin{array}{c}\text { Vaso-occlusive } \\
\text { Grave }\end{array}$ & & & & \\
\hline Infection sévère & 24 & 1 & 2 & 1 \\
\hline
\end{tabular}

Ces complications étaient majoritairement une anémie aigue (28 patients soit $22 \%$ ) et les infections sévères (28 patients soit $22 \%$ ). Un cas de séquestration splénique se compliquant d'une anémie aigue sévère a été noté chez un nourrisson. 
Les complications infectieuses étaient dominées par la pneumonie (21 cas soit $75 \%$ des infections) suivie de l'ostéomyélite aigue (6 cas soit 21,4 $\%)$; un cas de méningite d'origine tuberculeuse a été noté.

A côté de ces deux complications : nous avions enregistré quatre cas d'accident vasculaire cérébral (AVC), deux cas de syndrome thoracique aigue (STA) et un cas de priapisme chez un adolescent homozygote "SS ».

Durant leur suivi, neuf patients avaient présenté une complication chronique à type de lithiase biliaire chez 7 malades, d'ostéonécrose de la tête fémorale chez un patient et un cas d'hypersplénisme. Aucun cas d'ulcère de jambe ni d'insuffisance rénale chronique n’a été rapporté. L’âge minimum de survenue de ces complication chroniques était de 4 ans.

La fréquence des complications chroniques en fonction du profil des patients était représentée sur le tableau II.

Tableau II. Fréquences des complications chroniques en fonction du profil génotypique des patients

\begin{tabular}{lllll}
\hline \multicolumn{1}{c}{ Profil } & $\begin{array}{l}\text { Homozygote } \\
\text { SS }\end{array}$ & $\begin{array}{l}\text { Hétérozygote } \\
\text { SC }\end{array}$ & $\begin{array}{l}\text { Hétérozygote } \\
\text { SB plus }\end{array}$ & $\begin{array}{l}\text { Hétérozygote } \\
\text { SB zéro }\end{array}$ \\
\hline \hline Lithiase biliaire & $\mathbf{7}$ & $\mathbf{0 0}$ & $\mathbf{0 0}$ & $\mathbf{0 0}$ \\
Hypersplénisme & $\mathbf{1}$ & $\mathbf{0 0}$ & $\mathbf{0 0}$ & $\mathbf{0 0}$ \\
Ostéonécrose & $\mathbf{1}$ & $\mathbf{0 0}$ & $\mathbf{0 0}$ & $\mathbf{0 0}$ \\
Ulcèrede jambe & $\mathbf{0 0}$ & $\mathbf{0 0}$ & $\mathbf{0 0}$ & $\mathbf{0 0}$ \\
Insuffisance & $\mathbf{0 0}$ & $\mathbf{0 0}$ & $\mathbf{0 0}$ & $\mathbf{0 0}$ \\
cardiaque & & & $\mathbf{0 0}$ & $\mathbf{0 0}$ \\
$\begin{array}{l}\text { Insuffisance } \\
\text { rénale }\end{array}$ & $\mathbf{0 0}$ & $\mathbf{0}$ & & \\
\hline \hline
\end{tabular}

C.C : complications chroniques ; «SS » : drépanocytose homozygote « SS »; «SC » : drépanocytose « SC »; «SB » drépanocytose $\mathrm{S}$ béta thalassémie

Sur le plan biologique, le taux d'hémoglobine de base était défini comme le taux le plus constant sur les hémogrammes effectués en période de stabilité clinique. Ce taux moyen était de 8,6 g/dl avec un écart type de 1,4 chez les homozygotes SS, de $11 \mathrm{~g} / \mathrm{dl}$ (écart type 0,8 ) chez les sujets hétérozygotes SC et de 8,4 g/dl (écart type 2,3) dans le groupe des patients hétérozygotes $S \beta$.

Le nombre de transfusions (sang total ou concentrés de culot globulaire) par malade variait de 1 à 6 avec une moyenne de 1,8. Soixantesept $(53,2 \%)$ de nos patients avaient bénéficié au moins une transfusion sanguine.

Sur le plan thérapeutique, tous les patients de cette cohorte étaient sous acide folique à la dose de $5 \mathrm{mg}$ tous les deux jours. L'antibioprophylaxie par pénicilline $\mathrm{V}$ était systématiquement proposée aux enfants de moins de cinq ans; cependant, seuls $28 \%$ des patients recevaient régulièrement cette antibioprophylaxie. Quatre patient (3,1\%) ont été mis sous hydroxyurée en 
raison de la répétition des CVO et pour la prévention des AVC. Aucun patient n’a été dans un programme d'échange transfusionnel.

Quatre décès ont été enregistrés soit une létalité de 3,1 \%. Ces décès ont été liés à une infection sévère (chez deux patients), un STA (chez un malade) et le quatrième décès était secondaire à une anémie aigue.

\section{Discussion}

Dans toutes les études portant sur la drépanocytose, la forme homozygote SS est de loin la plus fréquente. Cependant nous avions noté une fréquence plus élevée de la forme hétérozygote SC comparée à la cohorte du Centre Hospitalier National d'enfants Albert Royer (CHNEAR) de Dakar au Sénégal (5,7\%) (Sall-Paye, 2015) et celle du Centre Hospitalier Abass Ndao (CHAN) de Dakar (Sénégal) (4,5\%) (Boiro et al., 2016). La fréquence de l’hémoglobine $C$ semble plus élevée dans la région Nord du Sénégal. Des études épidémiologiques basées sur le dépistage néonatal permettraient d'établir la cartographie des hémoglobinopathies.

L'âge de nos patients au moment de l'étude variait de deux ans cinq mois ( 2 ans 5 mois) à 19 ans 11 mois. La tranche d'âge (5-10 ans) était la plus représentée, soit 39,37 \%. Dans la cohorte du CHNEAR, qui regroupe le plus grand nombre de patients, Diagne et al. (2000) avaient également constaté un plus grand nombre de patients âgés de six à dix ans. Dans l'étude de DoumbiaDiarra (2009) au Mali, La tranche d’âge 0-4 ans était la plus représentée.

Les garçons étaient légèrement plus atteints que les filles avec un sexe ratio de 1,2.Cette prédominance masculine est observée dans de nombreuses études (Sall-Paye, 2015), (Diop et al., 2003), (Thiam et al., 2017) même si, rappelons que la transmission de la maladie n'est pas liée au sexe.

Le diagnostic de la drépanocytose survient tardivement avec un âge moyen au diagnostic qui était de 4 ans et 10 mois. Seuls $4 \%$ des patients avaient été diagnostiqués avant six mois. L’âge moyen au diagnostic était de 4 ans 11 mois au CHNEAR à Dakar (Sall-Paye, 2015), 4 ans et 4 mois au service de pédiatrie du CHAN (Boiro et al., 2016) et de de huit ans à Ziguinchor (Sénégal) (Thiam et al., 2017). Ce retard au diagnostic s’explique par l'absence d'un programme national de dépistage néonatal, la méconnaissance de la maladie par la population et le personnel de santé qui est souvent au premier contact dans les formations sanitaires de base et l'absence de moyen de diagnostic des hémoglobinopathies (hémogramme, électrophorèse de l'hémoglobine) dans les centres et postes de santé, voir dans certains hôpitaux régionaux. Le plus souvent le diagnostic est établi à l'occasion des manifestations cliniques de la maladie (crise vaso-occlusive (CVO), ictère, splénomégalie...) ou devant l'existence d'une anémie chronique. Avant l'âge de six mois, les manifestations cliniques de la maladie étant exceptionnelles contribuent davantage à retarder le diagnostic. Dans 
notre étude les circonstances de découvertes ont été une CVO dans 58,5 \% des cas et une anémie dans $21,1 \%$ des cas. Boiro et al. (2016) et Thiam et al. (2017) avaient également fait les mêmes observations.

Le suivi de la maladie débutait en moyenne à l'âge de 6 ans et 2 mois soit 16 mois après l'âge moyen au diagnostic. Ce décalage pourrait s'expliquer par le fait que les populations ont souvent recours en première intention aux traitements traditionnels. En effet, au Sénégal, comme dans la plupart des pays d'Afrique, environ $80 \%$ de la population a recours à la médecine traditionnelle pour les soins de santé primaires (Carboni, 2009).

Le faible niveau de revenus des ménages corrélé à leur faible niveau d'instruction peuvent également expliquer ce retard au diagnostic et à la prise en charge. En effet, près de la moitié des pères de familles (47,9 \%) et 2/3 des mères n'avaient pas d'emploi ou d'activités génératrices de revenus fixes. Dans les travaux de Boiro et al. (2016), 46,5 \% des patients étaient issus de ménages avec un niveau socio-économique bas. Chez Diagne et al. (2000), 30 $\%$ des pères avaient des revenus faibles et irréguliers voir nuls. En l'absence d'une couverture médicale, le coût moyen de la prise en charge d'un enfant drépanocytaire est estimé à cinq cent mille (500 000) francs par an au Sénégal. Le niveau d'instruction des parents était très faible, seuls $12,3 \%$ des pères et $4,3 \%$ des mères avaient reçu une instruction jusqu'au niveau secondaire correspondant au lycée.

La moitié de nos patients étaient issus d'un mariage consanguin dont 51,3 \% était apparenté au deuxième degrés. Ces données sont proches de celles de Boiro et al. (2016) et Abderrahim (2013) à Fès (Maroc) qui avaient retrouvé respectivement $50 \%$ et $57,1 \%$ de consanguinité parentale. Au Sénégal, et plus généralement en Afrique, les mariages consanguins occupent une place considérable dans les ménages.

Dans notre cohorte, la couverture vaccinale de 87,3\% par rapport aux vaccins du PEV est satisfaisante comparée aux taux de $78 \%$ et $65 \%$ rapportés respectivement par Boiro et al. (2016) et Diagne et al. (2000), ainsi qu'aux 72,7 \% rapportés par Gbadoé et al. ( 2001) au Togo. Quant aux vaccins fortement recommandés chez le sujet drépanocytaire, les taux de couverture sont encore faibles. Un quart de nos patients avaient reçu le vaccin polyosidique anti pneumococique et $44 \%$ étaient vaccinés contre la fièvre typhoïde. Chez Diagne et al. (2000), 21,4 \% des patients étaient protégés contre le pneumocoque et seuls $8,7 \%$ étaient vaccinés contre la fièvre typhoïde. Ces vaccins sont à la charge des patients et leurs coûts élevés expliquent les faibles pourcentages constatés.

La détermination du taux d'hémoglobine de base est un élément essentiel dans le suivi des malades drépanocytaires permettant de repérer les épisodes d'anémie aiguë. La transfusion sanguine est recommandée si le taux d'hémoglobine de base baisse d'au moins 2 g/dl (Médecins experts, 2018). 
Les drépanocytaires «SC » et « $\mathrm{S} \beta+$ » ont habituellement un taux d'hémoglobine de base plus élevé du fait d'une hémolyse chronique qui est moins importante que les drépanocytaires «SS » et " $\mathrm{S} \beta^{0}$ ». Les valeurs habituelles du taux d'hémoglobine de base chez les homozygotes "SS » se situent entre 6 et $10 \mathrm{~g} / \mathrm{dl}$ selon Begue \& Castello-herbreteau (2001) et Ferster et al. (2005). Dans notre cohorte, le taux d'hémoglobine de base était de 8,6 $\mathrm{g} / \mathrm{dl}$. Cette même valeur a été retrouvée dans les travaux de Thiam et al. (2017). Au CHAN, Boiro et al. (2016) avaient noté un taux d'hémoglobine moyen de 7,9 g/dl. Par contre au Gabon Thuillez et al. (1996) avaient obtenu un taux de base de $7 \mathrm{~g} / \mathrm{dl}$, en dessous de celui qui est observé habituellement au Sénégal. Les haplotypes "Arab-india » et "Sénégal » sont associés à une hémolyse moins importante et une expression clinique de la maladie moins sévère que les autres haplotypes. Des études réalisées chez les patients drépanocytaires sénégalais par Pagnier et al. (1984) montraient que $82 \%$ avaient un haplotype " Sénégal » au moins à l'état hétérozygote. Plus récemment, en 2018, sur une série plus importante, Doupa et al. (2018) avaient confirmé la présence de cet haplotype « Sénégal » chez $90 \%$ des patients sénégalais dont $58 \%$ à l'état homozygote.

La drépanocytose est une maladie chronique dont l'évolution peut être émaillée de complications aigues. Celles-ci sont très fréquentes dans les pays en développement et en font toute la gravité notamment les complications infectieuses et l'anémie aigue.

Les complications infectieuses sont à redouter particulièrement chez l'enfant du fait du risque important de décès en l'absence de prise en charge adéquate. L'incidence des bactériémies chez les enfants drépanocytaires serait 30 à 100 fois supérieure à celle observée chez les enfants du même âge indemnes de drépanocytose (Doumbia-Diarra, 2009). Vingt-huit cas soit $22 \%$ d'infections sévères ont été enregistrées dans notre étude. La pneumonie était la localisation infectieuse la plus fréquente. Au CHAN, Boiro et al (2016), avaient retrouvé 34 \% d'infections sévères dans sa cohorte, de même au Burkina-Fasso (Ye et al., 2008). Cette forte prévalence des infections pourrait s'expliquer par les mauvaises conditions d'hygiène, la faible couverture vaccinale notamment contre le pneumocoque, le méningocoque et une faible adhésion à l'antibioprophylaxie par pénicilline $\mathrm{V}$ chez les enfants de moins de cinq ans (28\% de nos patients recevaient réellement une antibioprophylaxie). L'anémie aigue était la deuxième complication aigue rencontrée dans notre série (22 \%). Elle est également une cause non négligeable de décès. Dans notre cohorte sur les quatre décès, l'un était secondaire à une anémie aigue. Chez Sall-Paye (2015), 22 \% des décès étaient secondaires à une anémie aigue. A Libreville dans l'étude de Koko et al. (1998), la létalité de l'anémie aigue était de $72 \%$. 
Parmi les accidents vaso-occlusifs majeurs nous avions notés quatre cas d'accident vasculaire cérébral (AVC) soit 3,1\%, deux cas de syndrome thoracique aigu (STA) et un cas de priapisme. Le risque d'AVC est une menace réelle qui pèse sur les enfants drépanocytaires, essentiellement chez les sujets «SS » et « $\mathrm{S} \beta^{\circ}$ ». Sa survenue peut être précoce. Une étude faite aux États-Unis par Kossorotoff et al. (2014) a permis d'évaluer le risque spontané d'AVC symptomatique à $11 \%$ avant l'âge de 20 ans chez les patients homozygotes "SS ». La prévalence des AVC était de $2 \%$ chez Sall- Paye (2015) , elle était plus élevée (5,1\%) chez Boiro et al. (2016). Le dépistage de la vasculopathie cérébrale drépanocytaire se fait au moyen d'un doppler transcranien annuel. Il est systématique à partir de deux ans, ce qui permet une stratification du risque (Médecins experts, 2018). Les enfants à risque élevé doivent bénéficier d'une prévention primaire par des échanges transfusionnels. Le STA, accident vaso-occlusif majeur, constitue une complication fréquente dans notre contexte ; sa létalité est également élevée. Au CHAN, Boiro et al. (2016) avaient noté quatre cas de STA. Un cas a été observé à Ziguinchor dans la cohorte de Thiam et al. (2017). Dans notre étude nous avions notifié deux cas. La survenue d'un STA chez un patient drépanocytaire est une indication de choix à un traitement par hydroxy-urée pour prévenir d'autres épisodes.

Les complications chroniques de la drépanocytose apparaissent dès l'enfance et leur fréquence a tendance à augmenter avec l'âge. Elles sont principalement liées à l'hémolyse chronique, l'ischémie et l'anémie. Les atteintes les plus fréquemment observées dans l'enfance sont la lithiase biliaire, l'ostéonécrose de la hanche, la rétinopathie et l'ulcère de jambe.

Dans notre cohorte, nous avions enregistré sept cas de lithiase biliaire, un cas d'ostéonécrose de la tête fémorale et un cas d'hypersplénisme. L'âge de survenue de ces complications variait de 4 à 16 ans. L'ostéonécrose de la tête fémorale peut survenir chez tous les variants génotypiques drépanocytaires mais est plus fréquente chez les patients $S \beta$ thalassémiques (Haute Autorité de Santé, 2005). L’hypersplénisme est une complication liée à la persistance de la splénomégalie chez le drépanocytaire (Diagne et al., 2010).

Dans notre cohorte, le nombre d'hospitalisations moyen au cours du suivi était de 1,9 avec des extrêmes de 1 à 10 . Parmi les patients suivis, 76 \% avaient été hospitalisés au moins une fois. Diagne et al. (2000) avaient retrouvé un nombre moyen d'hospitalisations de 2,4 chez les homozygotes « SS ». Plus de la moitié (53,2 \%) de nos patients avaient déjà bénéficié d'au moins une transfusion sanguine. Chez Diagne et al. (2000), le nombre de patients transfusés était de $30 \%$. Ce pourcentage élevé du nombre de transfusion dans notre série pourrait s'expliquer par une transfusion abusive avant la découverte de la maladie 
Nous avions enregistré une létalité de 3,1\%. Ce taux de décès est proche de celui décrit par Sall-Paye (2015) qui était de 2,3\%, en revanche il est plus élevé que celui décrit par Diagne et al. (2000) (1,1\%).

\section{Conclusion}

La drépanocytose est une maladie héréditaire de l'hémoglobine particulièrement fréquente en Afrique Noire. Elle constitue un réel problème de Santé publique du fait de son fort taux de mortalité notamment des syndromes drépanocytaires majeurs.

La forme homozygote est la plus fréquente (87 \% de nos patients), l'âge au diagnostic est encore tardif avec un âge moyen de 4 ans et 10 mois. La maladie est souvent découverte à l'occasion d'une CVO chez 58,5\% de nos patients ou devant une anémie dans $21,1 \%$ des cas. Il existe un décalage moyen de 16 mois entre le diagnostic et le début de la prise en charge. Le faible niveau d'instruction et le faible niveau socio-économique des ménages constatés dans notre étude pourraient l'expliquer. La couverture vaccinale par le PEV est satisfaisante dans notre cohorte $(87,4 \%)$ mais elle est encore très faible par rapport aux vaccins fortement recommandés chez le sujet drépanocytaire. Ce qui explique la fréquence élevée des complications infectieuses (22\%). L’anémie aigue était la deuxième complication aigue dans notre étude. Les complications chroniques sont dominées par la lithiase biliaire (sept cas). Les autres complications chroniques notamment rénales doivent être recherchées activement à partir de 10 ans. La létalité était de 3,1 $\%$ dans notre série.

Pour améliorer la prise en charge et le pronostic des malades, il sera nécessaire de :

- Mettre en place un programme national de dépistage néonatal

- Créer des centres de référence de prise en charge et de suivi des patients drépanocytaires

\section{References:}

1. Bardakjian J, Wajcman H. Epidémiologie de la drépanocytose. Rev Prat 2004 ; 54 : 1531-33.

2. Loupec RM. La drépanocytose. Observatoire régional de la santé en Guyanne. Octobre 2014.

3. Boiro D, Gueye M, Thiongane A, Ndongo AA, Houngbadji M, Keita Y, Dieng YJ, Seck N, Benjoloune A, Ndiaye O. Drépanocytose chez l'enfant : profils clinique et évolutif à propos de 138 cas suivis au service de pédiatrie de l'hôpital Abass Ndao de Dakar. Médecine d'Afrique noire 2016 ; 63, 6 : 327-32.

4. Diagne I, Ndiaye O, Moreira C, Signate-Sy H, Camara B, Diouf S, Diack-Mbaye A, Ba M, Sarr M, Sow D, Fall M. Les syndromes 
drépanocytaires majeurs en pédiatrie à Dakar (Sénégal). Arch Pédiatr $2000 ; 7: 16-24$.

5. North ML, Piffaut MC, Duwig I. Hémoglobinopathie : actualisation du diagnostic biologique. Revue Française des laboratoires 1995 ; 275 : 107-115.

6. Sall-Paye A. Létalite des syndromes drépanocytaires majeurs dans l'enfance et l'adolescence au sénégal : étude de la cohorte du Centre Hospitalier National d'Enfants Albert Royer de Dakar de 1991 à 2010. Thèse médecine université Cheikh Anta Diop de Dakar 2015 $\mathrm{n}^{\circ} 33$

7. Doumbia-Diarra A. Aspects épidémio-cliniques des enfants drépanocytaires suivis en pédiatrie de 2005 à 2008. Thèse médecine université de Bamako 2009.

8. Diop S, Mokono SO, Ndiaye M, Touré-Fall AO, Thiam D, Diakhaté L. Homozygous sickle cell in patients above 20 years age: follow-up of 108 patients in Dakar. La Revue de Médecine Interne 2003; 24: 711-715.

9. Thiam L, Dramé A, Coly I.Z, Diouf F.N, Seck N, Boiro D, Ndongo A.A, Basse I, Niang B, Ly-Deme I, Sylla A, Diagne I, Ndiaye O. Profils épidémiologiques, cliniques et hématologiques de la drépanocytose SS en phase intercritique chez l'enfant à Ziguinchor, Sénégal. Revue d’oncologie hématologie pédiatrique (2017) 5, 130135

10. Carboni C. La drépanocytose au Sénégal : un exemple de médecine traditionnelle. Thèse pharmacie Université de Grenoble 2009 nº 7046.

11. Abderrahim M. La drépanocytose chez l'enfant au service de pédiatrie à l'Hôpital Al Farabi Oujda. Thèse médecine université Sidi Mohammed Ben Abdelah de Fés 2013 nº 083/13.

12. Gbadoe AD, Atsou K, Agbodjan-Djossou OA, Tsolenyanu E, Nyadanu M. Prise en charge ambulatoire des drépanocytaires: évaluation de la première année de suivi des patients dans le service de pédiatrie de Lomé (Togo). Bull Soc Pathol Exo, 2001 ; 94,2 : 101-105.

13. Médecins experts. Guide de prise en charge : la drépanocytose en Afrique/ avec l'appui du gouvernement de la principauté de Monaco juin $2018: 29$

14. Begue $\mathrm{P}$, Castello-herbreteau B. La drépanocytose : de l'enfant à l'adolescent. Prise en charge en 2001. Bull Soc Pathol, Exo 2001 ; 94,2 : 85-89.

15. Ferster A, Kentos A, Bradstreet C, Vertongen F, Gulbis B. Drépanocytose : diagnostic et paramètres biologiques. JEUR 2005 ; 18 : 228-9. 
16. Thuilliez V, Ditsambou V, Mba JR, Mba-Meyo S, Kitengue J. Aspects actuels de la drépanocytose chez l'enfant au Gabon. Arch pédiatr 1996 ; 3 : 668-675.

17. Pagnier J, Mears JG, Dunda-Belkhodia O, Kim E, Shaefer-Rego KE, Beldjord C, Nagel RL, Labie D. Evidence for the multicentric origin of the sickle cell hemoglobin gene in Africa. Proc Natl Acad Sci USA 1984; 81: 1771-3.

18. Doupa D, Djité M, Kandji PM, Makalou D, Thiam S, Boye O et al. Polymorphism of the bête gene in homozygous sickle cell patients in Senegal and its influence on the main complications of the desease. Advances in Biochimistry 2018; 6, 3: 19-25

19. Ye D, Koueta F, Dao L, Kaboret S. Prise en charge de la drépanocytose en milieu pédiatrique : expérience du Centre Hospitalier Universitaire Pédiatrique Charles-De-Gaulle de Ouagadougou 2008 ; 18, 2.

20. Koko J, Dufillot D, Ba-Meyo JM, Gahouma D, Kani F. Mortalité des enfants drépanocytaires dans un service de pédiatrie en Afrique Central. Arch pédiatr 1998 ; 5 : 965-9.

21. Kossorotoff M, Grevent D, De montalembert M. cerebral vasculopathy in pediatric sickle-cell anemia. Arch pédiatr 2014; 21: 404-414

22. Haute Autorité de Santé. Prise en charge de la drépanocytose chez l'enfant et l'adolescent. Septembre 2005.

23. Diagne I, Diagne-Guèye NR, Fall AL, Déme I, Sylla A, Coly JI, Camara B, Sow HD. Epidemiology, and course of splenomegaly in children and adolescents with sickle cell disease in Sénegal. Arch pédiatr 2010; 17:1017-1025. 